\title{
AACR Annual Meeting 2014
}

\section{5-9 APRIL 2014}

San Diego Convention Center

San Diego, CA

Attendance: $>18,500$

The 105th Annual Meeting of the American Association for Cancer Research (AACR) brought together researchers, patient advocates and other cancer professionals from around the world to address the theme of "Harnessing Breakthroughs-Targeting Cures." The gathering in San Diego set a new attendance record, with a $2 \%$ increase in registered attendees over last year's conference in Washington, DC.

During the opening ceremony on Sunday morning, the AACR recognized several outstanding members of the cancer research community with its most prestigious awards. The Eighth Annual AACR Team Science Award went to the Malignant Brain Tumor Group, a collaboration between Duke University, Johns Hopkins University and the National Cancer Institute, led by Darell Doty Bigner (Duke University School of Medicine, Durham, NC). Douglas Hanahan (Swiss Federal Institute of Technology, Zurich) received the 11th Annual AACR Award for Lifetime Achievement in Cancer Research. The Eighth Annual AACR Margaret Foti Award for Leadership and Extraordinary Achievements in Cancer Research was presented to Webster K. Caveness (Ludwig Institute for Cancer Research, San Diego, CA). Elana Simon, a teenage cancer survivor, accepted the inaugural Young Champion in Cancer Research Award. After being diagnosed at 12 years old with fibrolamellar hepatocellular carcinoma, a rare liver cancer, Simon began genetic studies that helped to identify a specific fusion abnormality that may be associated with the disease. The abnormality is present in tumor tissue but not normal tissue samples from people with fibrolamellar hepatocellular carcinoma. Simon shared her story in a video presentation (available online at https://www.youtube.com/watch?v=GNSCk8JQ7Fw) and also spoke to attendees in person after accepting her award.

The award ceremony was immediately followed by the opening plenary session, which included presentations by Richard A. Young (Whitehead Institute for Biomedical Research, Cambridge, MA) on "Transcriptional and epigenetic control of tumor cells"; Carol L. Prives (Columbia University, New York, NY) on "The two faces of p53: Tumor suppressor and oncogene"; Louis M. Stadt (National Cancer Institute, Bethesda, MD) on "New lymphoma therapies based on functional and structural genomics"; Walter C. Willett (Harvard School of Public Health, Boston, MA) on "Diet and cancer: Status report in 2014"; and Roger M. Perlmutter (Merck

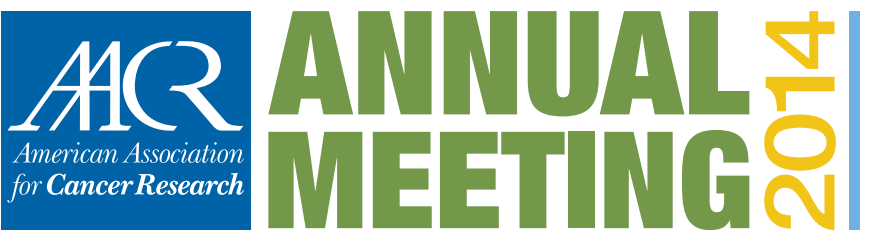

Research Laboratories, Rahway, NJ) on "Tumor-specific immune activation: Immuno-oncology comes of age." But perhaps the most anticipated part of the session was the presentation of the latest results from the PALOMA-1 trial, which evaluated combination therapy of palbociclib (a checkpoint inhibitor) and letrozole (an aromatase inhibitor) in $\mathrm{ER}^{+} \mathrm{HER} 2^{-}$metastatic breast cancer. Richard S. Finn (University of California Los Angeles) showed that "the palbociclib and letrozole combination demonstrated a significantly improved clinical outcome for patients who had hormone receptor-positive, metastatic breast cancer."

Special sessions on Monday focused on the Stand Up To Cancer (SU2C) initiative, an innovative program to accelerate research results by encouraging collaboration instead of competition among clinicians as well as pharmaceutical partners. Founded in 2008, it has funded more than 140 clinical trials enrolling over 4,000 patients and raised almost $\$ 270$ million for cancer research, in part by mobilizing prominent members of the entertainment industry to promote its mission. SU2C and the AACR work together to create and support interdisciplinary, multi-institutional, translational and clinical 'Dream Teams' dedicated to solving specific problems in cancer research. In the first session, representatives from the first five Dream Teams reported their 4-year results in presentations titled "Bringing epigenetic therapy to the forefront of cancer management," "Combining a PI3K inhibitor and PARP inhibitor in high grade serous ovarian cancer," "Understanding cancer metastasis through circulating tumor cells," "An integrated approach to targeting breast cancer molecular subtypes and their 'resistance' phenotypes" and "Cutting off the fuel supply: A new approach to the treatment of pancreatic cancer." The second session, led by SU2C Scientific Advisory Committee Chairperson and Nobel Laureate Phillip A. Sharp (Koch Institute for Integrative Cancer Research, Cambridge, MA), featured the introduction of new Dream Teams that will use targeted therapeutics and immunotherapy to improve patient outcomes.

Immunotherapy was big news throughout the meeting, with clinical trial results, case studies and translational investigations all highlighting the role of the immune system in oncogenesis and cancer treatment. Approaches that combine immunotherapy with existing or potential anticancer drugs seem poised to deliver benefits to patients, and researchers are actively investigating and developing these treatment strategies. 